\title{
The Influence of Network Density and Knowledge Inflow on Enterprise Management under the Innovative Concept
}

\author{
Rong Li \\ Kyung Hee University, Gyeonggi-do, Suwon-Si, South Korea, 449-701
}

Keywords: innovation concept; network density; knowledge inflow; enterprise management

\begin{abstract}
Under the conditions of macroeconomic regulation and control, the survival of enterprises is to improve the core competitiveness of enterprises. However, how to improve the competitiveness of enterprises has become a topic that we need to explore continuously in the process of operating enterprises. Today, the problem of enterprise management innovation is solved. The analysis of enterprise management competitiveness has been made. This paper first summarizes the enterprise management innovation, then analyzes the impact of network density on enterprise management innovation; it is essential to pay attention to the process of enterprise management innovation. The explanation is given, then some suggestions for enterprise management innovation are also given. Finally, this paper summarizes the skills and methods of enterprise management innovation.
\end{abstract}

\section{Introduction}

In the era of rapid development of the modern economy, how to get rapid development of enterprises is undoubtedly not a problem for business managers. In the management of enterprises, when conducting strategic decision-making management of enterprises, the control of the whole situation of the enterprise, the reasonable integration of the favorable resources of the major platforms, and finally the goal of improving the core competitiveness of the enterprise are all in order to make the enterprise fierce. There is a place to survive in the market competition.

Why is the competitiveness of enterprises more and more fierce under the current economic conditions? It is because more and more emerging enterprises, many companies are constantly closing down in the brutal market competition, and there is a very large replacement rate. So how to do it? It is very important for the company to develop and survive.

\section{Overview of Enterprise Management Innovation}

The comprehensive management of an enterprise can be subdivided into two categories, one is the objective category and the other is the subjective category. Innovation is the overall control of the enterprise, whether it is the control of the objective environment or the management of the structural elements of various departments within the enterprise. , all need innovation. New features of enterprise management include the estimation of risks, the sensitivity to new things, the exploitability of innovations, etc. The risk estimates are reflected in the weights of losses and losses that occur in the future development of the enterprise. Failure to make an accurate estimate will have unfortunate consequences for the company. The so-called sensitivity to new things means that new information existing in the outside world can be well utilized and processed. It can be well managed and used within the enterprise, and the new enterprise management system is constantly updated. Self-improvement of corporate management strategy decisions. The exploitability of the innovation point is well understood, that is, the timeliness of the innovation point and whether it can exert a powerful effect on the enterprise update management mechanism.

Innovation can be said to be the soul of management for enterprise management. Through the keen perception and observation of the market environment, and conforming to the objective development law of its existence, it can help enterprises to go into formalization, technology, technology, and new era. 


\section{The Impact of Network Density on Enterprise Management Innovation}

What is enterprise network density? It is the degree of communication and the degree of communication between the various industries and the industry. The greater the degree of communication and communication, the greater the density of the network. Therefore, the greater the density of communication and communication between the industry or cross-industry, the greater the density of the enterprise network, if the industry and industry between enterprises and enterprises The less the number of communication and exchanges through the network, the weaker the enterprise density. The final conclusion is that the network density plays a vital role in the management innovation of the enterprise.

A business decision maker must have a good understanding of the current state of network density. In the construction of the network density system, a decision made by a corporate decision-maker is not only directed at its own individual enterprise, but also involves the interests of stakeholders in the entire industry, the development of modern science and technology, the rapid changes in network density, we do Every decision made must be cautious, so when we make major strategic decisions, we are not a simple individual, but we are considering the same camp with other stakeholders.

The future direction of a company is also constrained by the density of the network. With the development trend of economic globalization in all walks of life, the development of enterprise management must conform to the needs of the entire market and finally balance the ecologicalization of the entire market. It further shows that enterprises and markets do not exist in isolation, and the lifeblood of the two is closely related. Therefore, when a company makes major decisions, it must comprehensively consider the macro environment of the entire market, and avoid making mistakes in decision-making. Therefore, many companies now have a variety of corporate management systems in order to adapt to this rapidly changing market.

The information flow between enterprises and enterprises, and the renewal and integration of network architecture are inseparable from network density. The more complex the network density, the more complex the relationship between business and business stakeholders. In order to protect their own interests, more and more corporate stakeholders have to communicate and communicate with the enterprise information between the industries. The management decisions are made quickly according to the acquired information, and appear in the process of obtaining information. A lot of complicated information circulation channels make many information not available to enterprise decision makers in time, which leads to the lag of a large amount of information transmission, which greatly affects the timely planning and execution of decision-making and development strategies of enterprise decision makers.

\section{The Impact of knowledge Inflow on Enterprise Management Innovation}

What is knowledge inflow? Many professionals and arguers give different answers to the definition of this term. Here are a few examples: The first so-called knowledge inflow refers to a process in which knowledge flows through a media channel. Mainly divided into formation, dissemination, involvement and innovation. The influx of the second knowledge includes a carrier's influence on the degree of knowledge absorption and application. The third knowledge itself is a carrier without boundaries, but it is more targeted for enterprises. Through the special needs of enterprises for knowledge, through the mining of themselves, we can find professional knowledge that can be valuable to the development of enterprises. It is the knowledge that it holds can be transformed into a business by processing.

Knowledge inflow can improve the company's knowledge level and social recognition as a whole. Knowledge inflow can also be used as a section of the enterprise network. The information held by a company is very limited. Only through the information exchange platform to absorb the knowledge of the external environment, in this way, the amount of knowledge reserves is continuously improved. Can improve the comprehensive technical capabilities of the enterprise.

In the process of knowledge integration, new areas of knowledge will be constantly discovered 
to increase productivity. Then create new knowledge flowing into the enterprise, the productivity level of the enterprise is to be improved by rubbing new sparks in different knowledge sections. In this way, continually injecting fresh blood into the enterprise plays a crucial role in reducing the homogenization of corporate decision-making.

In the process of knowledge inflow, the time cost of enterprise development is reduced, which provides a convenient channel for the development of enterprises. This is also an important factor to improve the productivity of enterprises.

The inflow of knowledge will also greatly improve the overall level of the enterprise. The quality of enterprise talent is the essence of a company's development. The accuracy and scientificity of the company's management system are also generated through knowledge inflow. A forward-thinking decision-maker needs the edification of new knowledge, and the decision-makers of the enterprise have a comprehensive level of enterprise. The level plays a very important role. So knowledge inflows are especially important for decision makers and corporate employees.

\section{Enterprise Management Innovation Needs Attention}

The enterprise management system must conform to the government's control measures. The rapid development of the modern economy, the existence of enterprise network density has become a normal state, the construction of network density can not be blindly obeyed, the volume of market accommodation is an important consideration, if poor management The impact on the market is extremely devastating. Therefore, it is necessary for the government to unite the market economic system, formulate scientific sustainability measures, and implement strict supervision on the network density order so that its development form can conform to the needs of the market, less media for information transmission, and reduce the occurrence of information transmission. The hysteresis, as soon as possible and accurate access to information, provides a good opportunity for the sound development of the market economy.

Under the economic system in which the country vigorously promotes self-employment, many enterprises have sprung up like mushrooms, all of which are benign effects brought by the market economy. At the same time, it is also a challenge for business managers. The lack of communication skills between enterprise managers and internal employees leads to the fault of corporate decision-making and the one-sidedness of information transmission. It is particularly difficult to form a corporate development process. The lack of enterprise management and the loopholes in the level of employee communication are difficult for the development of enterprises. Therefore, the development of a scientific enterprise employee function system structure to promote the accurate communication of information in all departments of the enterprise, accurate communication, the functional matching mechanism between departments and departments to make accurate management decisions according to this initiative, in order to improve the comprehensive management of enterprises from a macro perspective Level.

Knowledge transformation is a process of knowledge leapfrogging between industry and industry. Through the analysis of the core competitiveness of external knowledge, the company can beneficially absorb valuable parts to deepen and integrate, strengthen learning and extension in its own field, and fundamentally In order to achieve the connection of knowledge and control of the professional field, the ability of many enterprises to transform knowledge is also increasingly strengthened, and the development trend of liquidity of enterprise talents is becoming more and more obvious. The rotation system of posts is in the trend of liquefaction of such talents. It becomes familiar with the car. Therefore, the company must set up an ad hoc group to collect resources from the knowledge of the outside professional fields, and provide materials for the talents of the relevant attributes of the enterprise to improve their innovation ability. The transformation of knowledge and skills is the internal driving force for promoting the development of enterprises, and it is also fundamental.

Small and medium-sized enterprises in China occupy half of China's many enterprises, and it is also an important part of China's market economy macro-control. Therefore, the contradiction in the management of team-mate enterprise system construction is particularly prominent. Many small and 
medium-sized enterprises have very imperfect management systems. The organizational structure is also very simple, singular, and the staffing configuration is not scientific, resulting in the management level of China's small and medium-sized enterprises is generally not high. This status quo hinders the development of the enterprise, which in turn affects the quality of the entire market economy. It has become urgent to establish and improve the enterprise management system. Therefore, we will broaden the enterprise management information platform and increase the decision-making channels of enterprise managers, and quickly promote the strategic development of enterprises.

\section{Suggestions for Enterprise Management Innovation}

First, the management of network density and the reference of external knowledge of the network. The construction of network density can not blindly follow the trend, can not grasp the eyebrows and beards, combined with their specific circumstances to analyze, to a certain extent, the increase of network density can increase the source of information communication between enterprises. However, the increase in network density is also a double-edged sword. It also brings negative effects while the positive effects are presented. The scientificity, independence and communication speed of information will be affected by such negative effects. As a result of the direct decline in corporate management effectiveness, such negative effects, corporate managers and institutional regulators have taken relevant measures to balance the negative effects of excessive network density, not only to adjust the network density construction system as a whole. It is also necessary to accurately screen out high-quality information and clarify development policies and implementation strategies in light of the actual situation of the company itself.

Second, the choice of knowledge outside the network is also important. The sub-items involved in the professional field are also very complicated. The management of network density also requires the enterprise managers to have certain forward-looking capabilities. One-sided analysis of the immediate economic benefits brought by the network culture will damage the network density environment and thus lose the most. Good development time, the cognition of external knowledge structure also requires employees to review the situation, to control the overall situation, adjust the current problems encountered by the enterprise, and apply the social positive effects given by external knowledge to integrate into the enterprise. Plan the overall layout of the external knowledge of the network, formulate implementation guidelines in an orderly manner, and maximize the innovation ability of enterprise management. This is also inseparable from the efforts of every employee of the company.

Third, the richness of knowledge is fundamental to the development of a large enterprise. The source of innovation for enterprises is the introduction of new knowledge and the constantly updated market concept. How to promote the inflow of knowledge, the following suggestions are given: First, the improvement of the quality of talent. In the process of building the talent echelon of the human resources department of the enterprise, we must pay attention to the quality control. We must make professional choices and judgments on job seekers' work experience, professional technical ability, knowledge and skill reserve, and management experience. These new talents can bring new development atmosphere and hope to the enterprise through the reserve of their own knowledge. The second is the comprehensive quality of technology. Through the relevant cooperative relationship between enterprises and enterprises, there are technical exchanges, combined with the excellent management concepts and professional practical skills of other enterprises, through the clear insight into the market environment and the use of the development status of their own enterprises, it is also a Effective channels for technology introduction. Accurately grasping the timeliness of technical information greatly enhances the pace of business development.

The enterprise structure of small and medium-sized enterprises is very simple. Unlike large enterprises, the organizational structure of enterprises is linear and the functions are relatively weak. The phenomenon of enterprise gangs is very strict and the rights are seriously unbalanced. These small and medium-sized enterprises want to obtain A good information channel must actively 
explore, timely replenish the lost information, actively carry out academic exchanges between the same industry and different industries, participate in small and medium-sized enterprise management training sessions, and learn from and learn from the good development strategies and guidelines of large enterprises. Small and medium-sized enterprises have limited access to favorable development experience. They must put themselves into the market, participate in market exchanges, and make timely stops in places where they are insufficient, which will greatly reduce the enterprises themselves in the process of development. Obstacles to the arrival.

\section{Conclusion}

Network density and knowledge inflow are the top priorities of enterprise development and survival. As leaders and decision makers of enterprises, they should be regarded as a benchmark to improve management cognition. At the moment, the network density and the network density are monitored. Knowledge inflow has a far-reaching impact on the development of enterprises. At the same time, it is also necessary to clarify what is the basis of market economy conditions, accurately optimize the quality of talents, integrate the knowledge, and accurately control the network density. The enterprise benefits brought by technology have attracted enough attention, and the prospects of the market are far-sighted. Focusing on these factors will ultimately enable the company to be invincible.

\section{References}

[1] Zhang Songyi. The impact of network density and knowledge inflow on enterprise management innovation [J]. Enterprise Reform and Management. 2013

[2] Shi Liang. Network density, the impact of knowledge inflow on corporate management innovation [J]. Business Times. 2017

[3] Nie Fei. Analysis of the Influence of Network Density and Knowledge Inflow on Enterprise Management Innovation [J].Chinese Market.2017 